\title{
Special issue: The 7th International Symposium on Human Survivability “Let's Work Together Toward Achieving the Sustainable Development Goals"
}

\author{
Yuichi Ikeda ${ }^{1}$ \\ Published online: 10 February 2020 \\ (c) Japan Association for Evolutionary Economics 2020
}

The main aim of the 7th International Symposium on Human Survivability "Let's Work Together Toward Achieving the Sustainable Development Goals" was to bring together students and scholars in their latest academic research and knowledge to facilitate wider public discussion on solving global issues and moving toward achieving Sustainable Development Goals (SDGs). The organizer Graduate School of Advanced Integrated Studies in Human Survivability (GSAIS), also known as "Shishukan", holds once a year an international symposium that reflects on the global challenges facing humanity in areas such as energy, water, food, population, diseases, etc. For instance, the 2015 symposium focused on the global energy transformation, or the transition to sustainable sources of energy. The 2016 symposium discussed key issues related to "extreme", or "low-probability, high-impact" disasters. The 2017 symposium discussed how to bridge the gap between science and culture. In general, GSAIS/Shishukan international symposiums are an important stepping stone for the development of a holistic and transdisciplinary perspective on complex global issues that is also known as human survivability studies. The 2018 symposium takes an academic perspective on the global issues with an overarching concept of SDGs. Providing a fresh look, this symposium is created mostly by current students inspired by a mutually shared idea of working towards achieving SDGs.

SDGs are a universal call to action to end poverty, protect the planet and ensure that all people enjoy peace and prosperity. SDGs are a collection of 17 global goals set by the United Nations General Assembly in 2015. The SDGs cover social and economic development issues including poverty, hunger, health, education, global warming, gender equality, water, sanitation, energy, urbanization, environment and social justice. The goals are broad and interdependent, yet each has a separate list of targets to achieve. There are 169 targets for the 17 goals, all set to be achieved by

Yuichi Ikeda

ikeda.yuichi.2w@kyoto-u.ac.jp

1 Graduate School of Advanced Integrated Studies in Human Survivability, Kyoto University,

Sakyo-ku, Kyoto 606-8306, Japan 
2030. Achieving all 169 targets would signal accomplishing all 17 goals. Although, there is a general public consent that setting SDGs framework in 2015 was a milestone for humanity on its way to sustainable future there are a lot of skepticism as well. Sceptics are casting doubts on whether SDGs are actually achievable and whether they are really effective in encouraging humanity to switch from the "Business as Usual" development path. Furthermore, criticism is cast upon SDGs that some of the SDGs goals and targets might be contradictory to each other. Such negative relation between SDGs are often referend as "trade-offs". For example, seeking high levels of economic growth might undermine ecological, inequality reduction, among other sustainability objectives. Similarly, in the quest of increasing share of renewable energy to reduce GHG emissions, especially from transportation, global demand for biofuels surges, which raises fears of the potential destruction of wildlife habitats by being converted into farmland. On the other hand, there is a widespread consensus that progress in achieving some SDGs naturally leads to progress in achieving other SDGs. In other words, there are positive relationships between goals and targets, often referred as "co-benefits" or "synergies" between SDGs. For example, making significant progress in the availability of affordable education certainly contributes to reduction of poverty and hunger, brings about improvement of health and well-being among other benefits. Therefore, there is a pressing need to identify such relations between goals and targets and recognize their interactions to come up with appropriate solutions and strategies. Virtually all fields of natural and social sciences as well as humanities could be engaged in such task with both quantitative and qualitative research methods.

The 7th International Symposium on Human Survivability "Let's Work Together Toward Achieving the Sustainable Development Goals" was held on 10 December 2018 in Kyoto University. According to the arguments mentioned above the symposium aims at bringing together students and scholars in their latest academic research to facilitate a wider public discussion within the following scope:

(1) Original study focused on each of SDGs (with emphasis on trans-disciplinary studies).

(2) Synergy and trade-off between SDGs. Some of the goals can be achieved simultaneously, while others require compromise or higher-level solutions. Both quantitative and qualitative studies are included in the scope.

(3) Science communication for effective implementation of research results in society. It is crucial to bridge the gap between the academia and the society, or in other words, between the academic scientific research and implementation of such research results into society. In other words, how do we bring academic research results in to practice for the benefit of the society? How can we raise awareness of a wider public on the importance of such research? Even more generally, how can we promote United Nation's SDGs framework and the ideas behind it to a wider public?

All these issues were included in the scope of the symposium and were discussed between the participants including students, researchers, professors, professionals, representatives of international organizations, as well as general public, in a friendly 
and productive environment. The followings are a brief summary of major outcomes from the symposium.

The article "Vision, identity, and collective behavior change on pathways to sustainable futures", authored by Ilan Chabay pointed out that implementing the global aspirations embodied in the sustainable development goals of the United Nations will require societal transformation largely through collective behavior change at multiple geographic scales and governance levels across the world. Narrative expressions of visions of sustainable futures and narrative expressions of identity provide important, but underutilized insights for understanding affordances and obstacles to collective behavior change. Analyzing affective narrative expressions circulating in various communities seeking to implement aspects of sustainability opens up the opportunity to test whether affectively prioritized agent-based models can lead to novel emergent dynamics of social movements seeking sustainable futures.

The article "Measuring countries' progress on the Sustainable Development Goals: methodology and challenges", authored by Michal Shinwell proposed a methodology for measuring the distance countries must cover to reach the SDGs' targets by 2030, using publicly available data from official sources, for instance, databases developed by United Nations and Organization for Economic Co-operation and Development. The methodology allows comparison of performance across SDGs' goals, targets and indicators, and can provide a basis for further analysis, such as disaggregation, performance over time and forecasting achievements.

The article "Power grid with $100 \%$ renewable energy for small island developing states", authored by Yuichi Ikeda estimated system-wise levelized cost of electricity (LCOE) for a power grid with a high level of renewable energy using a grid optimization model. The estimated LCOE is as high as 397 \$MWh which is substantially higher than electricity prices for residential consumers in the US and Japan. The susceptibility analyses imply that the estimated LCOE increase of $223 \%$ with a $100 \%$ renewable power grid corresponds to an as high as $11 \%$ decrease in economic growth. The author suggested that the hydrogen production via the electrolysis of water using the excess energy supply from solar photovoltaics would reduce the LCOE and, therefore, higher economic growth would be attained with less $\mathrm{CO}_{2}$ emission.

The article "Coevolution of institutions and residents toward sustainable global development: a case study on the Kuni Umi solar power project on Awaji Island", authored by Natsuka Tokumaru regarded the program Kuni Umi Solar Power Plant with Resident Participation (KSPP) as an example of institutional change and demonstrated four perspectives. First, institutional change in Awaji has occurred with its rich social and natural capital resources and the crisis it has faced due to depopulation and a mega-earthquake as contrasting backdrops. Second, KSPP had an effective institutional design that integrates different actors- the feed-in-tariff system at a national level, the NPO Kuni Umi Association that builds and manages the KSPP, and the Awaji residents who financed the project. Third, Awaji residents were key micro-actors in supporting the KSPP project. Questionnaire survey shows that the initial period of living in Awaji significantly affects people's willingness to invest in socially responsible investments, whether for environmental protection or regional activation, because of their attachment to the region. Finally, KSPP is achieving 
renewable energy deployment and local activation as macro-outcomes of institutional change.

The article "Location-sector analysis of international profit shifting on a multilayer ownership-tax network", authored by Tembo Nakamoto, Odile Rouhban, and Yuichi Ikeda focused on treaty shopping among various ways to conduct international tax avoidance because tax revenues of developing countries have been heavily damaged through treaty shopping. The author constructed a multilayer ownershiptax network and proposed multilayer centrality that consider not only the value flowing in the ownership network but also the withholding tax rate. The analysis showed that firms in the sectors of Finance and Insurance and Wholesale and Retail trade etc. are involved with treaty shopping.

The article "The shift of food value through food banks: a case study in Kyoto, Japan", authored by Ayaka Nomura studied the diversion of food waste by food banks through an analysis of how the value of food is transformed by food banks and their recipients, from waste to things of value. The study was conducted through the observation of participants at food banks, food diaries, interviews with members of recipient organizations and final recipients, and a questionnaire with food assistance recipients in Kyoto, Japan. The first main point of this paper is the importance of food literacy to transform and optimize food donations into appreciated food, thereby avoiding food waste through the ingenuity of downstream actors. The second point is to uncover the shift of burden arising from the sorting, repurposing, and even discarding of the unusable portion of food donations. Above and beyond the burden of managing inconsistent and skewed food donations falls to recipient organizations and final recipients, the empirical data show that the recipient organizations and final recipients achieve this with very limited, scarce resources.

The article "An interdisciplinary study: disseminating information on dengue prevention and control in the world-famous travel destination, Bali, Indonesia", authored by Minako Jen Yoshikawa, Rita Kusriastuti, and Christina Liew carried out a study comprising fieldwork and literature analyses, to address the proposed empowerment of hotels to disseminate information on dengue prevention and control to international travelers. The fieldwork included an outreach workshop and questionnaire, conducted by partnering the local health authorities and hotel association. The questionnaire revealed that only $67 \%$ of the participating hotel personnel know that dengue is mosquito-borne. $83 \%$ of the facilities reported mosquito nuisance problems, and fogging was the most utilized control measure of choice. The analyses indicate that there is a role for the hotel sector in promoting anti-dengue awareness and activities in Bali. Such an initiative could assist Bali to work towards the good health and well-being for the sustainable development goals.

The workshop was supported by the discretionary expense of the president of Kyoto University, "Souchou Sairyou Kiehi". I am grateful for the members of the student organizing committee: Mr. Ryou Tsuchida, Ms. Ayaka Watanabe, Ms. Shoko Iwasaki, Mr. Myasoedov Fedor and Mr. Tomoki Hanabusa, and for the other members of the faculty organizing committee: Professor Kaoru Takara, Professor Kaoru Sekiyama, Associate Professor Takashi Kanamura, Associate Professor Marc-Henri Deroche, Program-Specific Professor Hiroaki Takashima, and Program-Specific Associate Professor Tomonaga Yoshida. We wish to express our thanks to the 
support of Professor Yuji Aruka (Chuo University), the staff members of Springer Japan, and the members of the conference secretariat in GSAIS, Kyoto University. Finally, we would like to thank all authors for their contributions to this special feature of Evolutionary and Institutional Economics Review.

Publisher's Note Springer Nature remains neutral with regard to jurisdictional claims in published maps and institutional affiliations. 